\title{
Measurement of the Tau Lepton Lifetime
}

\author{
CLEO Collaboration
}

Submitted to Physics Letters B 
CLNS 96/1417

CLEO 96-8

\section{Measurement of the Tau Lepton Lifetime}

R. Balest, ${ }^{1}$ B.H. Behrens,${ }^{1}$ K. Cho, ${ }^{1}$ M. Daoudi,${ }^{1}$ W.T. Ford,${ }^{1}$ M. Lohner,${ }^{1}$ H. Park, ${ }^{1}$ P. Rankin, ${ }^{1}$ J. Roy,${ }^{1}$ J.G. Smith,${ }^{1}$ J.P. Alexander,${ }^{2}$ C. Bebek,${ }^{2}$ B.E. Berger,${ }^{2}$ K. Berkelman, ${ }^{2}$ K. Bloom, ${ }^{2}$ D.G. Cassel, ${ }^{2}$ H.A. Cho, ${ }^{2}$ D.M. Coffman, ${ }^{2}$ D.S. Crowcroft,${ }^{2}$ M. Dickson, ${ }^{2}$ P.S. Drell, ${ }^{2}$ D.J. Dumas,${ }^{2}$ R. Ehrlich,${ }^{2}$ R. Elia, ${ }^{2}$ P. Gaidarev,${ }^{2}$ R.S. Galik,${ }^{2}$

B. Gittelman, ${ }^{2}$ S.W. Gray, ${ }^{2}$ D.L. Hartill, ${ }^{2}$ B.K. Heltsley, ${ }^{2}$ C.D. Jones, ${ }^{2}$ S.L. Jones, ${ }^{2}$ J. Kandaswamy, ${ }^{2}$ N. Katayama, ${ }^{2}$ P.C. Kim, ${ }^{2}$ D.L. Kreinick, ${ }^{2}$ T. Lee, ${ }^{2}$ Y. Liu, ${ }^{2}$ G.S. Ludwig, ${ }^{2}$ J. Masui, ${ }^{2}$ J. Mevissen ${ }^{2}$ N.B. Mistry, ${ }^{2}$ C.R. ${ }^{2},{ }^{2}$ E. Nordberg, ${ }^{2}$ J.R. Patterson, ${ }^{2}$ D. Peterson, ${ }^{2}$ D. Riley, ${ }^{2}$ A. Soffer, ${ }^{2}$ C. Ward,${ }^{2}$ P. Avery,${ }^{3}$ C. Prescott,${ }^{3}$ S. Yang, ${ }^{3}$ J. Yelton,${ }^{3}$ G. Brandenburg, ${ }^{4}$ R.A. Briere, ${ }^{4}$ T. Liu, ${ }^{4}$ M. Saulnier, ${ }^{4}$ R. Wilson, ${ }^{4}$ H. Yamamoto, ${ }^{4}$ T. E. Browder,${ }^{5}$ F. Li,${ }^{5}$ J. L. Rodriguez ${ }^{5}$ T. Bergfeld, ${ }^{6}$ B.I. Eisenstein,${ }^{6}$ J. Ernst, ${ }^{6}$ G.E. Gladding, ${ }^{6}$ G.D. Gollin, ${ }^{6}$ I. Karliner, ${ }^{6}$ M. Palmer, ${ }^{6}$ M. Selen, ${ }^{6}$ J.J. Thaler, ${ }^{6}$

K.W. Edwards ${ }^{7}$ K.W. McLean, ${ }^{7}$ M. Ogg, ${ }^{7}$ A. Bellerive, ${ }^{8}$ D.I. Britton, ${ }^{8}$ R. Janicek, ${ }^{8}$

D.B. MacFarlane, ${ }^{8}$ P.M. Patel, ${ }^{8}$ B. Spaan,${ }^{8}$ A.J. Sadoff, ${ }^{9}$ R. Ammar,${ }^{10}$ P. Baringer, ${ }^{10}$ A. Bean,${ }^{10}$ D. Besson,${ }^{10}$ D. Coppage,${ }^{10}$ N. Copty,${ }^{10}$ R. Davis,${ }^{10}$ N. Hancock, ${ }^{10}$ S. Kotov,${ }^{10}$ I. Kravchenko, ${ }^{10}$ N. Kwak, ${ }^{10}$ S.Anderson, ${ }^{11}$ Y. Kubota, ${ }^{11}$ M. Lattery, ${ }^{11}$ J.J. O'Neill, ${ }^{11}$ S. Patton, ${ }^{11}$ R. Poling, ${ }^{11}$ T. Riehle, ${ }^{11}$ A. Smith, ${ }^{11}$ V. Savinov, ${ }^{11}$ M.S. Alam, ${ }^{12}$ S.B. Athar, ${ }^{12}$

I.J. Kim, ${ }^{12}$ Z. Ling, ${ }^{12}$ A.H. Mahmood, ${ }^{12}$ H. Severini, ${ }^{12}$ C.R. Sun, ${ }^{12}$ S. Timm, ${ }^{12}$ F. Wappler, ${ }^{12}$ J.E. Duboscq ${ }^{13}$ R. Fulton, ${ }^{13}$ D. Fujino, ${ }^{13}$ K.K. Gan,${ }^{13}$ K. Honscheid, ${ }^{13}$ H. Kagan, ${ }^{13}$ R. Kass,${ }^{13}$ J. Lee ${ }^{13}$ M. Sung, ${ }^{13}$ A. Undrus, ${ }^{13 *}$ C. White,${ }^{13}$ R. Wanke, ${ }^{13}$ A. Wolf, ${ }^{13}$ M.M. Zoeller, ${ }^{13}$ B. Nemati, ${ }^{14}$ S.J. Richichi, ${ }^{14}$ W.R. Ross, ${ }^{14}$ P. Skubic,${ }^{14}$ M. Wood, ${ }^{14}$ M. Bishai, ${ }^{15}$ J. Fast,${ }^{15}$ E. Gerndt,${ }^{15}$ J.W. Hinson, ${ }^{15}$ D.H. Miller, ${ }^{15}$ E.I. Shibata, ${ }^{15}$ I.P.J. Shipsey ${ }^{15}$ M. Yurko, ${ }^{15}$ L. Gibbons, ${ }^{16}$ S.D. Johnson, ${ }^{16}$ Y. Kwon, ${ }^{16}$ S. Roberts, ${ }^{16}$ E.H. Thorndike, ${ }^{16}$ C.P. Jessop, ${ }^{17}$ K. Lingel, ${ }^{17}$ H. Marsiske, ${ }^{17}$ M.L. Perl, ${ }^{17}$ S.F. Schaffner, ${ }^{17}$ R. Wang, ${ }^{17}$ T.E. Coan,${ }^{18}$ V. Fadeyev, ${ }^{18}$ I. Korolkov, ${ }^{18}$ Y. Maravin, ${ }^{18}$ I. Narsky, ${ }^{18}$ V. Shelkov, ${ }^{18}$ R. Stroynowski, ${ }^{18}$ J. Staeck, ${ }^{18}$ I. Volobouev, ${ }^{18}$ J. Ye, ${ }^{18}$ M. Artuso, ${ }^{19}$ A. Efimov, ${ }^{19}$ M. Gao, ${ }^{19}$ M. Goldberg, ${ }^{19}$ R. Greene, ${ }^{19}$ D. He, ${ }^{19}$ S. Kopp,${ }^{19}$ G.C. Moneti, ${ }^{19}$ R. Mountain, ${ }^{19}$ Y. Mukhin, ${ }^{19}$ T. Skwarnicki, ${ }^{19}$ S. Stone, ${ }^{19}$ X. Xing, ${ }^{19}$ J. Bartelt,${ }^{20}$ S.E. Csorna,${ }^{20}$ V. Jain,${ }^{20}$ S. Marka, ${ }^{20}$ A. Freyberger,${ }^{21}$ D. Gibaut,${ }^{21}$ K. Kinoshita, ${ }^{21}$ I.C. Lai, ${ }^{21}$ P. Pomianowski, ${ }^{21}$ S. Schrenk, ${ }^{21}$ G. Bonvicini, ${ }^{22}$ D. Cinabro, ${ }^{22}$

B. Barish, ${ }^{23}$ M. Chadha, ${ }^{23}$ S. Chan, ${ }^{23}$ G. Eigen, ${ }^{23}$ J.S. Miller,${ }^{23}$ C. O'Grady, ${ }^{23}$

M. Schmidtler, ${ }^{23}$ J. Urheim, ${ }^{23}$ A.J. Weinstein, ${ }^{23}$ F. Würthwein, ${ }^{23}$ D.M. Asner, ${ }^{24}$ M. Athanas, ${ }^{24}$ D.W. Bliss, ${ }^{24}$ W.S. Brower, ${ }^{24}$ G. Masek, ${ }^{24}$ H.P. Paar, ${ }^{24}$ J. Gronberg, ${ }^{25}$ C.M. Korte ${ }^{25}$ D.J. Lange, ${ }^{25}$ R. Kutschke ${ }^{25}$ S. Menary, ${ }^{25}$ R.J. Morrison, ${ }^{25}$ S. Nakanishi, ${ }^{25}$ H.N. Nelson, ${ }^{25}$ T.K. Nelson, ${ }^{25}$ C. Qiao,${ }^{25}$ J.D. Richman, ${ }^{25}$ D. Roberts, ${ }^{25}$ A. Ryd,${ }^{25}$ H. Tajima, ${ }^{25}$ and M.S. Witherell ${ }^{25}$

(CLEO Collaboration) 
${ }^{1}$ University of Colorado, Boulder, Colorado 80309-0390

${ }^{2}$ Cornell University, Ithaca, New York 14853

${ }^{3}$ University of Florida, Gainesville, Florida 32611

${ }^{4}$ Harvard University, Cambridge, Massachusetts 02138

${ }^{5}$ University of Hawaii at Manoa, Honolulu, HI 96822

${ }^{6}$ University of Illinois, Champaign-Urbana, Illinois, 61801

${ }^{7}$ Carleton University, Ottawa, Ontario K1S 5B6 and the Institute of Particle Physics, Canada

${ }^{8}$ McGill University, Montréal, Québec H3A 2 T8 and the Institute of Particle Physics, Canada

${ }^{9}$ Ithaca College, Ithaca, New York 14850

${ }^{10}$ University of Kansas, Lawrence, Kansas 66045

${ }^{11}$ University of Minnesota, Minneapolis, Minnesota 55455

${ }^{12}$ State University of New York at Albany, Albany, New York 12222

${ }^{13}$ Ohio State University, Columbus, Ohio, 43210

${ }^{14}$ University of Oklahoma, Norman, Oklahoma 73019

${ }^{15}$ Purdue University, West Lafayette, Indiana 47907

${ }^{16}$ University of Rochester, Rochester, New York 14627

${ }^{17}$ Stanford Linear Accelerator Center, Stanford University, Stanford, California, 94309

${ }^{18}$ Southern Methodist University, Dallas, Texas 75275

${ }^{19}$ Syracuse University, Syracuse, New York 13244

${ }^{20}$ Vanderbilt University, Nashville, Tennessee 37235

${ }^{21}$ Virginia Polytechnic Institute and State University, Blacksburg, Virginia 24061

${ }^{22}$ Wayne State University, Detroit, Michigan 48202

${ }^{23}$ California Institute of Technology, Pasadena, California 91125

${ }^{24}$ University of California, San Diego, La Jolla, California 92093

${ }^{25}$ University of California, Santa Barbara, California 93106

(June 28, 1996)

\begin{abstract}
We measure the $\tau$ lepton lifetime with $\tau^{+} \tau^{-}$pairs in which one or both of the $\tau$ 's decays to three charged particles. The data were collected with the CLEO II detector operating at the electron-positron collider CESR at energies on and near the $\Upsilon(4 S)$. We use displacements of the three-track vertices to determine the $\tau$ lifetime. The result is $\tau_{\tau}=289.0 \pm 2.8 \pm 4.0 \mathrm{fs}$.
\end{abstract}

*Permanent address: BINP, RU-630090 Novosibirsk, Russia 


\section{INTRODUCTION}

The decay of the $\tau$ lepton provides a useful testing ground for the Standard Model of electroweak interactions [1]. Within the framework of this model the $\tau$ is a sequential lepton, and therefore its properties such as mass, lifetime, and leptonic decay rate are related to each other. In particular, its coupling to the $W$ is the same as that of the $\mu$, and its lifetime is related to the muon lifetime:

$$
\tau_{\tau}=\tau_{\mu}\left(m_{\mu} / m_{\tau}\right)^{5} \mathcal{B}\left(\tau^{-} \rightarrow e^{-} \nu_{\tau} \bar{\nu}_{e}\right)\left(1-\delta_{r}\right)
$$

The calculated $\tau$ lifetime $\tau_{\tau}$ depends directly on experimental measurements of the muon mass $m_{\mu}$ and lifetime $\tau_{\mu}$, and of the $\tau$ mass $m_{\tau}$ and electronic branching fraction $\mathcal{B}\left(\tau^{-} \rightarrow\right.$ $\left.e^{-} \nu_{\tau} \bar{\nu}_{e}\right)$. The term $\delta_{r}$ represents the radiative correction along with the contribution of the electron mass to the phase space factors; it has the calculated value 0.0004 [2]. Using the world average values for the measured quantities [3,4] we find the predicted lifetime to be:

$$
\tau_{\tau}=(1.632 \pm 0.0012) \times 10^{-12} B\left(\tau^{-} \rightarrow e^{-} \nu_{\tau} \bar{\nu}_{e}\right)=294 \pm 3 \text { fs }\left(294 \times 10^{-15} \mathrm{~s}\right) .
$$

In this paper we present a new measurement of the $\tau$ lifetime based on a high statistics sample of tau pairs produced in $e^{+} e^{-}$annihilations. We reconstruct vertices from decays with three charged tracks to measure the decay point, in events with the other tau decaying into either one ( 1 vs 3 ) or three charged tracks ( 3 vs 3 ). With the 3 vs 3 sample we use both decay vertices without reference to the production point, which is uncertain because of the beam size. This is the first high statistics measurement by this technique, which will be extendible to future experiments having precision vertex detection and high event rates.

\section{INSTRUMENTATION}

The data were accumulated at the Cornell Electron-positron Storage Ring (CESR). The sample corresponds to a total integrated luminosity of $3 \mathrm{fb}^{-1}\left(3.6 \mathrm{fb}^{-1}\right)$ used for the $1 \mathrm{vs} 3$ (3 vs 3 ) analysis, with approximately two thirds of the data collected at the $\Upsilon(4 S)$ (centerof-mass energy $E_{\mathrm{cm}}=10.58 \mathrm{GeV}$ ), and the rest at energies near the resonance. These luminosities correspond to the production of $2.7 \times 10^{6}\left(3.4 \times 10^{6}\right) \tau$-pairs, of which $25 \%$ are of the 1 vs 3 and $1 \%$ of the 3 vs 3 topologies [3]. We include events with additional neutral pions in the 1 vs 3 subsample, but not in the 3 vs 3 subsample.

The CLEO II detector [5] emphasizes precision charged particle tracking and high resolution electromagnetic calorimetry. The detector elements surround a $3.5 \mathrm{~cm}$ radius beryllium beam pipe which presents $0.44 \%$ of a radiation length of material at normal incidence. Charged particle tracking is accomplished with the use of information from three concentric wire drift chambers: a 6 layer straw tube chamber (PT), with innermost layer located 4.7 $\mathrm{cm}$ from the interaction point, a 10 layer vertex drift chamber (VD), and a large volume drift chamber (DR) of 51 layers (40 axial and 11 stereo). The $z$ position (coordinate along the beam axis) is determined from the DR stereo layers and from cathode strips located on

the inner and outer walls of both the VD and DR. For charged particle momentum analysis a superconducting coil supplies a 1.5 Tesla magnetic field throughout the tracking volume. 
Surrounding the tracking system inside the solenoid is an electromagnetic calorimeter containing $7800 \mathrm{CsI}(\mathrm{Tl})$ crystals. The calorimeter provides high quality photon detection, $\pi^{0}$ reconstruction, and electron identification.

\section{EVENT SELECTION}

We select 1 vs 3 tau pairs [6] by accepting events which have 4 charged tracks, with a net charge of zero. To ensure that the event is well measured, we demand that each track's point of closest approach to the beam axis have perpendicular distance (DCA) less than $1 \mathrm{~cm}$ and $z$-displacement from the interaction point less than $10 \mathrm{~cm}$. We define two hemispheres separated by the plane perpendicular to the highest momentum charged track. One hemisphere must have one charged track, and the other must have 3 charged tracks. QED backgrounds such as radiative Bhabha and two-photon interactions are suppressed by requiring that the total energy of the event be greater than $0.30 E_{\mathrm{cm}}$, the total shower energy be less than $0.75 E_{\mathrm{cm}}$, and at most one track be identified as an electron. The invariant mass of charged and neutral particles within each hemisphere must be less than $1.6 \mathrm{GeV}$ (assuming all charged tracks are pions), and the missing mass of the event must be between 0.5 and $7.0 \mathrm{GeV}$; these cuts reduce $q \bar{q}$ and two-photon backgrounds. The total momentum vector of the particles in each hemisphere is required to point to the barrel region of the detector, $|\cos \theta|<0.80$, where the polar angle $\theta$ is defined with respect to the beam direction. With Monte Carlo events [7] we determine the selection efficiency to be $10.2 \%$.

The selection of double 3 -prong events is discussed in detail in Ref. [8]. We require six charged tracks, three in each hemisphere bounded by the plane perpendicular to the chargedparticle thrust axis. The net charge in each hemisphere is required to be \pm 1 and the total charge of the event must be zero. Each track must have $|\cos \theta|<0.81$, and momentum $p$ greater than $0.05 E_{\mathrm{b}}$, where $E_{\mathrm{b}}=\frac{1}{2} E_{\mathrm{cm}}$ is the beam energy. To eliminate secondary decays such as $K_{S} \rightarrow \pi^{+} \pi^{-}$, we veto events if there are any tracks with DCA greater than $1.5 \mathrm{~cm}$. We reject events if an identified electron, when paired with another track, is consistent with arising from a photon conversion. We suppress $q \bar{q}$ background and feed-across from other $\tau$ decay modes by vetoing events with calorimeter showers which have energy greater than $100 \mathrm{MeV}$, are more than $30 \mathrm{~cm}$ from the nearest hadronic charged track, and have a lateral profile consistent with that of photons. Events containing showers with energy greater than $800 \mathrm{MeV}$ are rejected regardless of the shower location and shape. Background from $q \bar{q}$ events is reduced further by requiring both $3 \pi$ invariant masses to be less than $1.5 \mathrm{GeV}$. To reject two-photon background, we require that the polar angle of the missing momentum satisfy $\left|\cos \theta_{\text {miss }}\right|<0.98$ and that the scalar sum of the momenta of the six tracks be at least $0.45 E_{\mathrm{cm}}$.

An event of either topology must satisfy further requirements to ensure track quality. Two of the three tracks comprising a vertex must have $0.3<p<4.0 \mathrm{GeV} / c$, at least 39 drift chamber layers contributing to the track fit, DCA less than $5 \mathrm{~mm}$, and average track residual less than $300 \mu \mathrm{m}$. For each 3 -prong cluster in the 1 vs 3 ( 3 vs 3 ) events, all three (two of three) tracks must have at least 2 PT hits, 4 VD hits, and 10 DR hits. Events are rejected if the 3 -prong vertex reconstruction code cannot fit the tracks to a common vertex, or if the fit $\chi^{2}$ exceeds 24 (for 1 degree of freedom). Finally, all remaining events must have 
a measured value for $c \tau$ between -4110 and $4290 \mu \mathrm{m}$ and a measured $c \tau$ uncertainty of less than $400 \mu \mathrm{m}$. The final data sample contains 553201 vs 3 and 21593 vs 3 events.

\section{BACKGROUND ESTIMATES}

We model the remaining hadronic background in the sample by Monte Carlo generated $q \bar{q}[9]$ and $B \bar{B}[10]$ events processed through a simulation of the detector [11].

We estimate the amount of $q \bar{q}$ background in the 1 vs 3 sample, using both data and simulations, to be $1.3 \pm 0.3 \%$. We calculate the two-photon background to be less than $0.5 \%$. By varying the selection criteria and studying the data and Monte Carlo we estimate the remaining background levels from Bhabha and $\mu$-pair events with a photon conversion in the beam pipe to be less than $0.2 \%$. We have also investigated possible contamination from beam gas interactions and $\Upsilon(4 S) \rightarrow B \bar{B}$ decays and found the contributions of these sources to be negligible $(0.02 \pm 0.1 \%$ and $0.15 \pm 0.15 \%$ respectively $)$.

For the 3 vs 3 analysis, where $q \bar{q}$ background is more significant, we scale the Monte Carlo estimate by $1.2 \pm 0.2$ to agree with data in regions where $q \bar{q}$ background dominates. We estimate the two-photon contribution from distributions sensitive to this background, and $\Upsilon(4 \mathrm{~S})$ background from Monte Carlo. The resulting estimates are $5.5 \pm 1.3 \% q \bar{q},<0.1 \%$ $B \bar{B}$ and $<0.2 \%$ two-photon events.

\section{LIFETIME DETERMINATION}

The tau proper flight distance, $c \tau$, is calculated from:

$$
c \tau=\frac{L}{\gamma \beta}=\frac{m_{\tau}}{p_{\tau}} \frac{L_{x y}}{\sin \theta}
$$

where $L$ is the decay length, and $\gamma, \beta$, and the magnitude of the $\tau$ 's momentum $p_{\tau}$ are calculated from the beam energy. Initial state radiation reduces the $\tau$ energy somewhat; from the simulation we find the correction to the average decay distance to be $3.1 \%$ from this effect for our sample. $L_{x y}=L \sin \theta$ is the component of the flight path in the precision measurement projection, transverse to the $z$ axis. We determine the $\tau$ polar angle $\theta$ from the combined vector momentum of the three charged tracks.

\section{A. Vertex reconstruction}

For each $\tau$ we determine the most probable projected decay length $L_{x y}$ from the equation

$$
L_{x y}=\frac{X t_{x} \sigma_{y}^{2}+Y t_{y} \sigma_{x}^{2}-\left(X t_{y}+Y t_{x}\right) \sigma_{x y}}{t_{y}^{2} \sigma_{x}^{2}+t_{x}^{2} \sigma_{y}^{2}-2 t_{x} t_{y} \sigma_{x y}}
$$

with $X=X_{v}-X_{b}, Y=Y_{v}-Y_{b}$ for the 1 vs 3 events, and $X=\frac{1}{2}\left(X_{v 2}-X_{v 1}\right), Y=\frac{1}{2}\left(Y_{v 2}-Y_{v 1}\right)$ for the 3 vs 3 events. In these equations, $\left(X_{v}, Y_{v}\right)$ are the transverse decay coordinates of the $\tau$ decay point and $\left(X_{b}, Y_{b}\right)$ are the corresponding coordinates of its production point. The determination of these quantities is discussed below. In Eq. $4, \sigma_{x}^{2}, \sigma_{y}^{2}$, and $\sigma_{x y}$ are 
elements of the error matrix for $(X, Y)$. Finally, $t_{x}$ and $t_{y}$ are direction cosines of the threeprong momentum vector (of the momentum-difference vector for 3 vs 3 events). This vector is our approximation to the flight direction of the $\tau$ 's. From the Monte Carlo calculation we find that the distribution of angles between the true and approximated tau directions caused by omission of the undetected neutrino has a mean of zero and an rms deviation of $5^{\circ}$. Negative decay distances arise when the reconstructed vertex lies in the hemisphere opposite that of the 3 -prong momentum vector. We determine $\left(X_{v}, Y_{v}\right)$ for each vertex with a $\chi^{2}$ minimization algorithm $[12,13]$ which constrains the three charged tracks to come from a common point.

\section{B. Beam positions}

Beam positions $\left(X_{b}, Y_{b}\right)$, required for the 1 vs 3 measurement, are determined with hadronic events for each data run. For this purpose we select events that have more than four charged tracks to exclude most $\tau$ pairs. The resulting sample for a typical run contains about 350 events. Track quality cuts eliminate poorly fit and low momentum tracks which might have large multiple scattering effects. We fit tracks to a common vertex with the same algorithm as that used for finding the $\tau$ decay point, iterating with exclusion of any outlying tracks. We determine an average position and rms deviation for each data run. The uncertainties in the average values of $\left(X_{b}, Y_{b}\right)$ are typically $35 \mu \mathrm{m}$ and $15 \mu \mathrm{m}$, respectively. The full error on the production point also includes a contribution from the finite extent of the beams $(350 \mu \mathrm{m}$ in $x$ and $10 \mu \mathrm{m}$ in $y)$.

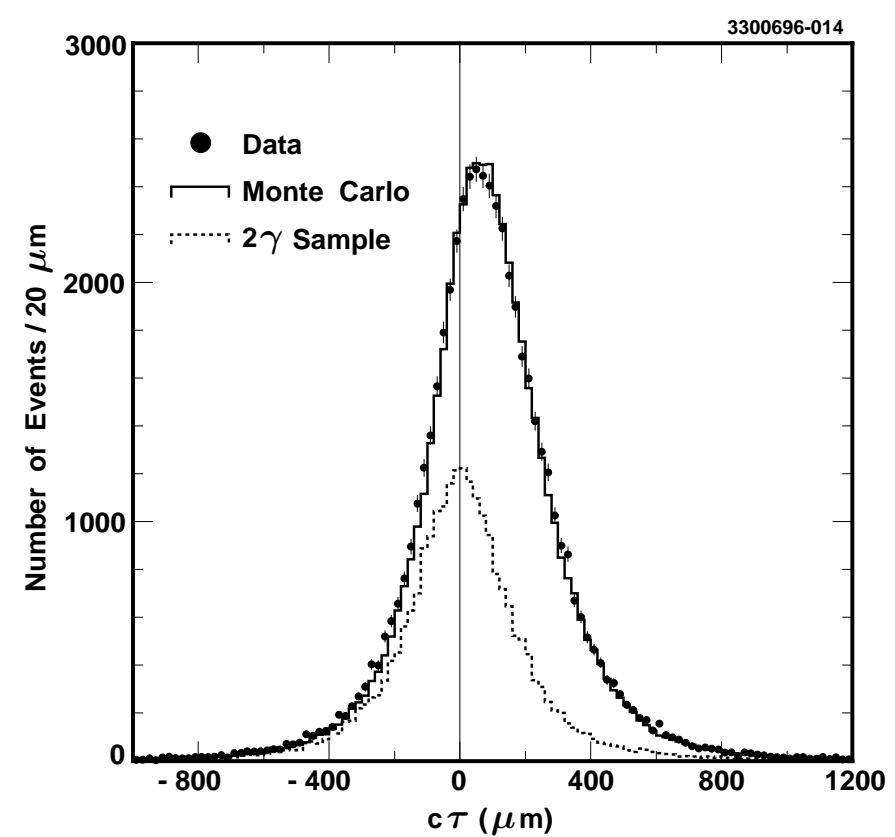

FIG. 1. Decay length distribution for Monte Carlo and Data for 1 vs 3 events. The dashed histogram is for the control sample, discussed in the text, of two-photon events with four charged tracks in the final state. 


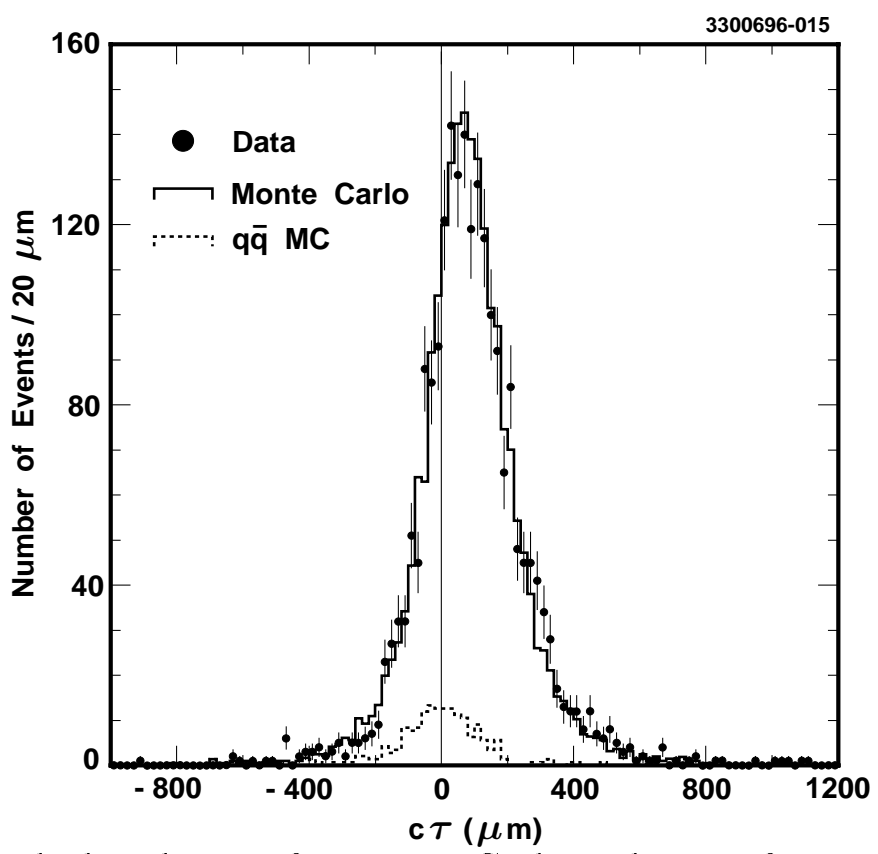

FIG. 2. Decay length distribution for Monte Carlo and Data for 3 vs 3 events. Data are indicated by points with error bars, simulation (signal plus background) by the solid histogram, and $q \bar{q}$ background simulation by the dashed histogram.

\section{Lifetime calculation}

Distributions of the measured $c \tau$ calculated according to Eq. 3 for 1 vs 3 and 3 vs 3 events are shown in Figs. 1 and 2, respectively. Also displayed are Monte Carlo calculations (including contributions from the backgrounds), showing good agreement with the shape of the data distributions. We determine the lifetime from weighted averages $c \tau_{\text {meas }}$ of these distributions, obtaining the weight of each event from the vertex fit error matrix. We prefer this averaging procedure to a fitting technique (e.g. maximum likelihood) because it is less sensitive to modeling of the resolution. The mean value of the distribution is independent of the scale of the decay length errors. We describe our tests for bias in Sect. VI below.

The mean $\tau$ lifetime, $\tau_{\tau}$, and the mean lifetime of the event sample, $\tau_{\text {meas }}$, are related by:

$$
\tau_{\text {meas }}=\left(1-f_{b g}\right) s_{c o r r} \tau_{\tau}+f_{b g} \tau_{b g}
$$

where $f_{b g}$ is the fraction of non- $\tau$ events in the sample and $\tau_{b g}$ is their mean lifetime. The correction factor $s_{\text {corr }}$ reflects the effects of initial-state radiation, the imperfectly known $\tau$ flight direction, and any vertex reconstruction bias (see below). Effectively $s_{\text {corr }}$ is calculated as the ratio of reconstructed to generated lifetime from the Monte Carlo, and assumes the value $0.977 \pm 0.003(1.005 \pm 0.017)$ for the 1 vs $3(3 \mathrm{vs} 3)$ measurement.

We compute the lifetime of the background sample from a Monte Carlo simulation, confirmed with data. For the hadronic background in the 1 vs 3 ( 3 vs 3 ) sample we find $c \tau_{b g}=12 \pm 8 \mu \mathrm{m}(1.7 \pm 7.2 \mu \mathrm{m})$.

We measure $c \tau_{\text {meas }}$ to be $83.02 \pm 0.85 \mu \mathrm{m}$ for the $1 \mathrm{vs} 3$ sample and $87.8 \pm 3.1 \mu \mathrm{m}$ for the 3 vs 3 sample. After correcting for backgrounds, initial state radiation, and bias (Eq. 5), we find $c \tau_{\tau}$ to be $86.22 \pm 0.88 \mu \mathrm{m}$ for the $1 \mathrm{vs} 3$ sample and $92.5 \pm 3.2 \mu \mathrm{m}$ for the $3 \mathrm{vs} 3$ sample. 


\section{CONSISTENCY CHECKS AND SYSTEMATIC ERRORS}

To check for internal consistency we have examined the sensitivity of our results to: separation of the data into sets for different run periods, delimited for example by a change of the gas mixture (argon/ethane to DME) in the PT; number of PT hits included on the tracks; vertex fit $\chi^{2} /$ DoF; and for the 1 vs 3 sample, sign of charge of the contributing $\tau$ and the decay mode of the $\operatorname{tag} \tau$. No significant variations were observed, indicating no bias within $0.5 \mu \mathrm{m}$.

We used Monte Carlo calculations to perform detailed checks for bias, varying inputs such as $\tau_{\tau}$, detector resolution, simulation of particle interactions in the detector, and the vertex fitting algorithm. We estimate biases from the vertex determination (included in $s_{\text {corr }}$, Eq. 5) of $+0.5 \pm 0.4 \mu \mathrm{m}$ for both the 1 vs 3 and 3 vs 3 sample.

As a further check for bias, we performed a test with a large sample of two-photon events with four charged tracks, reconstructed as for 1 vs 3 tau pairs. The decay length distribution for these events is included in Fig. 1. For this reaction we expect a mean decay length of zero. After correcting for contamination of this sample we measure an average decay length of $-2.2 \pm 1.5 \mu \mathrm{m}$, confirming that we have no large bias. We conservatively assign a systematic error of $1.1 \mu \mathrm{m}$ to account for effects due to tracking and vertexing, and $0.3 \mu \mathrm{m}$ for the method of extracting the mean decay length for the 1 vs 3 analysis.

We have studied the sensitivity of the 1 vs 3 lifetime measurement to the beam position and size by independently shifting the assumed values of both in data and Monte Carlo samples. Variation of the beam position or size by $100 \mu \mathrm{m}$ does not shift the central lifetime value; we assign $0.2 \mu \mathrm{m}$ as the beam related systematic error.

For the 3 vs 3 analysis, vertex measurement systematic error estimates include the finite statistics of the $\tau$ Monte Carlo sample $(1.5 \mu \mathrm{m})$, biases in the vertex reconstruction algorithm $(0.6 \mu \mathrm{m})$, the track reconstruction $(0.3 \mu \mathrm{m})$, and the technique for extraction of the lifetime from the distribution $(1.5 \mu \mathrm{m})$. The last was estimated by comparing the weighted mean $c \tau$ with an unweighted trimmed mean for a range of trim fractions. The combined systematic error from these sources is $2.2 \mu \mathrm{m}$.

The systematic error due to the uncertainty in the background fraction is calculated to be $0.3(1.3) \mu \mathrm{m}$ for the 1 vs 3 (3 vs 3 ) analysis, taking into account uncertainties in the modeling of the background composition and the sensitivity of the background fraction to the event selection criteria. This error also accounts for the change in $f_{b g}$ when we use two independent methods to estimate it. Finally, the systematic error due to the uncertainty in the lifetime of the background is calculated to be $0.1(0.8) \mu \mathrm{m}$ for the 1 vs 3 (3 vs 3 ) analysis.

Systematic errors for the two measurements are summarized in Table I. The total systematic error for each analysis is obtained by combining the contributions in quadrature. Thus for the 1 vs 3 sample we measure $c \tau_{\tau}=86.2 \pm 0.9 \pm 1.2 \mu \mathrm{m}$, with the first error statistical and the second systematic. For the 3 vs 3 sample we measure $c \tau_{\tau}=92.5 \pm 3.2 \pm 2.7 \mu \mathrm{m}$. 
TABLE I. Systematic errors on $c \tau$ for both analyses in $\mu \mathrm{m}$.

\begin{tabular}{lcc}
\hline \hline Source & 1 vs 3 & 3 vs 3 \\
\hline Tracking and Vertexing & 1.1 & 1.6 \\
Lifetime Extraction from Distribution & 0.3 & 1.5 \\
Beam Position + Size & 0.2 & 0.0 \\
Background Fraction & 0.3 & 1.3 \\
Background Lifetime & 0.1 & 0.8 \\
\hline Total & 1.20 & 2.7 \\
\hline \hline
\end{tabular}

\section{CONCLUSIONS}

Using a large sample of 1 vs $3 \tau$ events we measure $\tau_{\tau}=287.6 \pm 2.9 \pm 4.0$ fs. With an independent sample of 3 vs $3 \tau$ events we find $\tau_{\tau}=309 \pm 11 \pm 9$ fs. Taking account of the common systematic error of $3.3 \mathrm{fs}$, associated mainly with the track reconstruction, we find the combined result from both samples

$$
\tau_{\tau}=289.0 \pm 2.8 \pm 4.0 \mathrm{fs} .
$$

This is consistent with other measurements of $\tau_{\tau}$, such as recently published results from $Z^{0}$ decay $\tau_{\tau}=297 \pm 9 \pm 5$ fs [14], $291.4 \pm 3.0$ fs [15], $289.2 \pm 1.7 \pm 1.2$ fs [16], and $293.7 \pm 2.7 \pm 1.6$ fs [17]. Our result also agrees with the Standard Model prediction given in Eq. 2.

\section{ACKNOWLEDGEMENTS}

We gratefully acknowledge the effort of the CESR staff in providing us with excellent luminosity and running conditions. J.P.A., J.R.P., and I.P.J.S. thank the NYI program of the NSF, M.S. thanks the PFF program of the NSF, G.E. thanks the Heisenberg Foundation, K.K.G., M.S., H.N.N., T.S., and H.Y. thank the OJI program of DOE, J.R.P., K.H., and M.S. thank the A.P. Sloan Foundation, and A.W. and R.W. thank the Alexander von Humboldt Stiftung for support. This work was supported by the National Science Foundation, the U.S. Department of Energy, and the Natural Sciences and Engineering Research Council of Canada. 


\section{REFERENCES}

[1] S. L. Glashow, A Salam, S. Weinberg, Rev. Mod. Phys 52, (1980) 515.

[2] W. J. Marciano and A. Sirlin, Phys. Rev. Letters 61 (1988) 1815.

[3] L. Montanet et al. (Particle Data Group), Phys. Rev. D 50 (1994) 1173.

[4] J. Z. Bai et al. (BES collaboration), Phys. Rev. D53 (1996) 20.

[5] Y. Kubota et al. (CLEO Collaboration), Nucl Inst. Meth. 320 (1992) 66.

[6] D. Bortoletto et al. (CLEO Collaboration), Phys. Rev. Lett. 71 (1993) 1791.

[7] Koralb (v.2.2)/Taudla (v.2.4): S. Jadach and Z. Was, Comput. Phys. Commun. 64 (1991) 267; S. Jadach, J. H. Kühn, and Z. Was, ibid., 76 (1993) 361.

[8] R. Balest et al. (CLEO Collaboration), Phys. Rev. Letters 75 (1995) 3809.

[9] JETSET 7. 3: T. Sjöstrand and M. Bengtsson, Comput. Phys. Commun. 43 (1987) 367.

[10] P. Avery, K. Read and G. Trahern, CLEO internal note CSN-212.

[11] GEANT 3.15: R. Brun et al., CERN DD/EE/84-1. We have checked that our results are insensitive to the photon energy cutoff used in the simulation.

[12] J. Whitmore, Ph.D. thesis, Ohio State University (1992) unpublished.

[13] W. T. Ford, CLEO internal note CSN 94/329 (1994).

[14] K. Abe et al. (SLD collaboration), Phys. Rev. D52 (1995) 4828.

[15] P. Abreu et al. (DELPHI collaboration), Physics Letters B365 (1996) 448.

[16] G. Alexander et al. (OPAL Collaboration), Phys. Lett. B374 (1996) 341.

[17] D. Buskulic et al. (ALEPH Collaboration), CERN-PPE/95-128 (1995). 